\title{
TYPE IA SUPERNOVAE
}

\author{
J. CRAIG WHEELER \\ Department of Astronomy \\ University of Texas \\ Austin, Texas 78712 \\ U.S.A.
}

\begin{abstract}
Spectral calculations show that a model based on the thermonuclear explosion of a degenerate carbon/oxygen white dwarf provides excellent agreement with observations of Type Ia supernovae. Identification of suitable evolutionary progenitors remains a severe problem. General problems with estimation of supernova rates are outlined and the origin of Type Ia supernovae from double degenerate systems are discussed in the context of new rates of explosion per $\mathrm{H}$ band luminosity, the lack of observed candidates, and the likely presence of $\mathrm{H}$ in the vicinity of some SN Ia events. Re-examination of the problems of triggering Type Ia by accretion of hydrogen from a companion shows that there may be an avenue involving cataclysmic variables, especially if extreme hibernation occurs. Novae may channel accreting white dwarfs to a unique locus in accretion rate/mass space. Systems that undergo secular evolution to higher mass transfer rates could lead to just the conditions necessary for a Type Ia explosion. Tests involving fluorescence or absorption in a surrounding circumstellar medium and the detection of hydrogen stripped from a companion, which should appear at low velocity inside the white dwarf ejecta, are suggested. Possible observational confirmation of the former is described.
\end{abstract}

\section{Introduction}

While there is broad general acceptance of the notion that Type Ia supernovae, SN Ia, occur as a result of interacting binary stars, there is very little direct evidence. The most solid aspect of SN Ia is the conclusion that they result from the explosion of a carbon/oxygen white dwarf. Harkness (1991a) has shown that a particular subsonic deflagration model results in a composition-velocity distribution that gives an excellent agreement between the theoretical spectra near maximum light and the observations which show iron and the products of carbon and oxygen burning. Refinements to the atmosphere models serve to increase the agreement (Harkness 1991b). Other plausible deflagration models do not give such good agreement, so the spectral constraints are very strong. While other means of exploding the white dwarf involving a mixture of deflagrations and supersonic detonations are still being explored (Khokhlov 1991; Woosley 1991), it is very difficult to see how any initial configuration except a degenerate carbon/oxygen white dwarf could possibly provide an adequate progenitor structure. 
The question of how a carbon/oxygen white dwarf gets to its explosive endpoint is still very unclear. This review will summarize general issues concerning supernova rates in $\S 2$, some new constraints on double degenerate progenitors in $\S 3$, reconsider the possible role of cataclysmic binaries in $\S 4$, and describe some general observational tests for various types of binary evolution in $\S 5$. A summary is given in $\S 6$. Other aspects of these problems are given in Wheeler (1990), Wheeler and Harkness (1990), and Wheeler (1992) and references therein.

\section{Supernova Rates}

There are fundamental questions about the proper way to analyze supernova rates in galaxies of different types. It is well known that supernova rates per galaxy are not meaningful because galaxies vary too much in mass, luminosity, and evolutionary history. One seeks, rather, some means to normalize with respect to these variations and determine a measure of the underlying progenitor population. As described in van den Bergh and Tammann (1991), an obvious parameter to consider is the mass of the galaxy. Since the mass of the galaxy is not known directly, the blue luminosity has been used as a convenient integral quantity with which to normalize supernova rates.

This procedure may be fundamentally flawed. It is not clear, for instance, that mass is the proper normalizing quantity. Does one mean the luminous mass, the dark mass, the gas mass? Different mass components may determine different supernova rates, e.g. Type I verses Type II, in different ways. Normalizing with respect to the blue luminosity, as a convenient substitute for the mass, is also suspect. The blue luminosity measures the A star population of a galaxy. This population is relevant to neither Type II supernovae that come from more massive stars, nor to Type Ia that probably come from a more complex binary evolution that may not be necessarily related to any single mass range or photometric measure. The very fact that the mass/luminosity ratio varies among galaxies of different morphological type means that caution must be exercised in interpreting the data in the way it is traditionally presented. Van den Bergh and Tammann give the SN Ia rate per unit blue luminosity a factor of two higher in E galaxies than in Sc galaxies. The rate for Type II supernovae is three times higher in Sc galaxies than in Sb galaxies and, of course, the rate of SN II remains unobservably small in E galaxies. This is prima facie evidence that the blue luminosity does not single out the common underlying progenitor population of either SN Ia or SN II.

Van den Bergh (1990) has recently presented an interesting variation on this theme. He noted that if an older population dominated by red giants were the common progenitor population among $\mathrm{Sc}$ and $\mathrm{E}$ galaxies, then the $\mathrm{H}$ band luminosity of those galaxies that is dominated by the red giant population might be a more proper measure of the progenitor population. Van den Bergh thus computed rates of SN Ia per unit $\mathrm{H}$ band luminosity for various morphological types. The somewhat surprising result was that the discrepancy between the E and Sc galaxies was even larger! The rate of SN Ia explosions per unit $\mathrm{H}$ luminosity was ten times larger for Sc's than for E's. Van den Bergh interprets this as meaning that his assumption was in error in the sense that the $\mathrm{H}$ luminosity was too small a quantity by which to divide, and that there must be a larger luminosity contributing to the SN Ia progenitor population in Sc's. Dividing by that larger luminosity would then give a smaller rate per unit luminosity for the Sc's and hence more nearly the same quantity for both $\mathrm{E}$ and Sc galaxies. Van den Bergh's conclusion is that there must be a significant younger population contributing to the SN Ia rate. 
Van den Bergh's analysis may illustrate deeper problems. Note that the basis for this analysis (and all others) is that there is some total rate of supernovae in a galaxy of a given morphological type, $R_{T}=R_{T}\left(L_{B}, L_{H}\right)$. The rate of supernova explosions per unit luminosity in the $B$ and $H$ bands is then $r_{B}=R_{T} / L_{B}$ and $r_{H}=R_{T} / L_{H}$, respectively. Note that this makes no assumption about the functional form of $R_{T}$. It specifically does not need to be linear in either $L_{B}$ or $L_{H}$. This basic formulation does require

$$
\frac{\mathrm{r}_{\mathrm{B}}}{\mathrm{r}_{\mathrm{H}}}=\frac{\mathrm{L}_{\mathrm{H}}}{\mathrm{L}_{\mathrm{B}}}
$$

This means that one can write an expression comparing the rates $\mathrm{rE}_{\mathrm{E}}$ and $\mathrm{rS}_{\mathrm{S}}$ in $\mathrm{E}$ and $\mathrm{S}$ galaxies, respectively, as follows:

$$
\frac{\mathrm{r}_{\mathrm{B}, S} \mathrm{r}_{\mathrm{H}, \mathrm{E}}}{\mathrm{r}_{\mathrm{B}, \mathrm{E}} \mathrm{r}_{\mathrm{H}, \mathrm{S}}}=\frac{\left(\mathrm{L}_{\mathrm{H}} / \mathrm{L}_{\mathrm{B}}\right) \mathrm{S}}{\left(\mathrm{L}_{\mathrm{H}} / \mathrm{L}_{\mathrm{B}}\right) \mathrm{E}}
$$

From van den Bergh and Tammann (1991) $\mathrm{r}_{\mathrm{B}, \mathrm{S}} / \mathrm{r}_{\mathrm{B}, \mathrm{E}} \sim 0.5$ and from van den Bergh (1990), $\mathrm{r}_{\mathrm{H}, \mathrm{E}} / \mathrm{r}_{\mathrm{H}, \mathrm{S}} \sim 0.1$ whereas $\left(\mathrm{L}_{\mathrm{H}} / \mathrm{L}_{\mathrm{B}}\right) \mathrm{S} \sim 6.3$ and $\left(\mathrm{L}_{\mathrm{H}} / \mathrm{L}_{\mathrm{B}}\right) \mathrm{E} \sim 25$. Thus the left hand side of equation 2 gives $\sim 0.05$ and the right hand side gives $\sim 0.25$. The relative data on $S$ and $E$ galaxies is thus inconsistent by a factor of about 5 . While the rates are still rather uncertain because of poor statistics, this discrepancy may be related to a flaw in the logic that leads one to write equation 1 . That is, no supernova rate per unit luminosity in a single band may be the proper measure of normalized supernova rates. Further analysis of this problem seems worthwhile. In the meantime, van den Bergh's work does suggest that SN Ia require a significant, if not uniquely, young progenitor population in spirals. Whether the same implication holds for ellipticals can not be addressed given the inconsistency of equation 2 .

\section{Constraints on Double Degenerate Progenitors}

It is interesting to examine this new constraint provided by van den Bergh (1990) on particular models for the origin of SN Ia. Although it is basically appealing, the idea that SN Ia are caused to explode by transferring mass from a red giant or other more or less normal companion has been difficult to quantify for a variety of reasons (see next section). This was a major impetus behind consideration of double degenerate progenitors which would not involve the transfer of hydrogen, but helium or carbon/oxygen (Iben and Tutukov 1984; Webbink 1984). In this picture, the time scale for explosion is set by the evolution of the progenitor stars, and, even more significantly, by the gravitational radiation time scale for the two white dwarfs to spiral together after being left in moderately close orbit by common envelope evolution. The double degenerate mechanism can then, in principle, provide progenitors with a range of progenitor lifetimes from a single generation of stars. The common envelope ejection process is not well known in detail, but happens roughly when the binding energy of the two white dwarf cores is comparable to the binding energy of the common envelope. This means that the two cores can not get arbitrarily close before the envelope is ejected and hence that there is a lower limit to the subsequent gravitational radiation time scale. If this is sufficiently short, then the progenitor lifetime can be rather small and set by the time scale of the previous evolutionary phases. Since the progenitor population is that of moderately massive stars, less than about $8 \mathrm{M}_{\odot}$, they are rather short lived, 
variables. Standard estimates of their number density (Patterson 1984) tend to show that there are too few to account for the explosion of SN Ia, independent of details of their evolution. On the other hand as reported here by Bond, the rate of birth of double planetary nebula nuclei, the presumed ancestors of cataclysmic variables, is one in 10. This is sufficient to give a much larger reservoir of cataclysmic variables than estimated by Patterson The notion of deep hibernation (Shara et al. 1986) has been shunned recently by some of the authors of that concept, but the basic idea supports the notion that there could be many low luminosity cataclysmic variables that escape standard detection techniques. The density of novae could thus be as high as given by Bath and Shaviv (1978) although not, as noted by Wheeler (1992), for the reasons they give.

Given that there might be far more cataclysmic variables than thought, there is still the question of whether they can reach an explosive endpoint. Wheeler (1992) outlines a possible scenario that turns nova explosions to a possible advantage. He notes that if nova explosions remove mass from a white dwarf, then it will evolve to a locus, the "nova/dud" line, below which explosions are not possible. For low mass white dwarfs, below the nova/dud line, the mass that is transferred to the white dwarf will tend to stick and the white dwarf will grow in mass. This suggests that all nova-like systems, indeed all cataclysmic variables will tend to converge to the nova/dud line, as illustrated schematically in Figure 1. The subsequent secular evolution of the

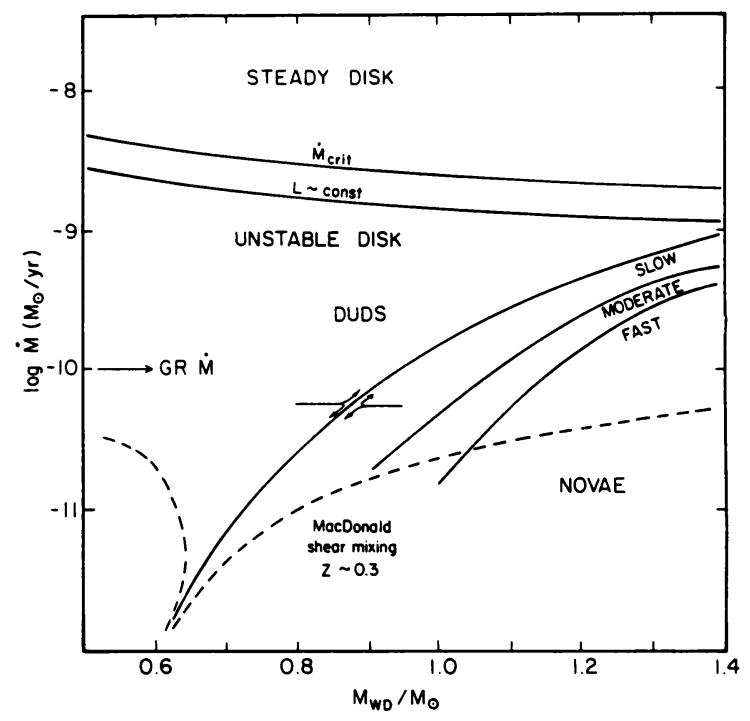

Figure 1. Systematics of the "novae/dud" line as a function of mass transfer rate, white dwarf mass, and metal enrichment as given by Wheeler (1992). Systems to the left of the line will add mass to the white dwarf and systems to the right of the line will tend to strip mass from the white dwarf, leading to a tendency to converge to the nova/dud locus. Dashed lines show the boundary above which the convergence will take less than a Hubble time if the accretion and stripping are very efficient. The nearly horizontal lines represent transfer rates above which the disk is stable. 
but expected to survive long enough to escape the spiral arms in which they were born, another necessary constraint (Maza and van den Bergh 1976). Qualitatively, then, the double degenerate mechanism satisfies the van den Bergh constraint that some proportion of the progenitors be rather short lived compared to a Hubble time.

The other obvious constraint on the double degenerate mechanism is the existence of progenitor systems. Although close double degenerates of small mass are known, searches for double degenerates among the DA white dwarfs in the orbital range up to 10 hours have been unsuccessful (Robinson and Shafter 1989: Bragaglia, et al. 1990; Foss, Wade, and Green, 1991). Some double degenerates of larger orbital period have been found (Saffer, Liebert, and Olszewski, 1988; Bragaglia, et al. 1990), but the time scale for decay by gravitational radiation exceeds the Hubble time. Foss, Wade, and Green argue that there can not be enough hot, young double degenerates to make a substantial contribution to the rate of explosion of SN Ia. A significant population of double degenerates that are born at larger orbital separation and have had time to cool and escape detection could exist, but would necessarily have long lifetimes, in violation of the van den Bergh constraint. On the other hand, Iben and Tutukov (1992) argue that given the sensitivity of the orbital decay time scale to the orbital period,

$$
\tau_{\mathrm{GW}}=10^{7.4} \mathrm{yr} \mathrm{P}^{8 / 3}\left(\mathrm{M} / \mathrm{M}_{\odot}\right)^{-5 / 3},
$$

Foss et al. should have only seen one system in 80 , not inconsistent with the zero in 40 systems they report.

Thus while double degenerates may in principle give young progenitors, it is not clear that there are sufficient hot progenitors to account for the rate of SN Ia. Note that if there are fundamental problems in talking about SN Ia rates per unit luminosity, it is not clear that one knows how to quantitatively determine whether sufficient progenitor systems are observed. It is in any case of interest to examine other possible progenitor systems and to seek observational constraints on various progenitors, including double degenerates.

\section{Reconsideration of the Possible Role of Cataclysmic Binaries}

There are a number of quantitative problems with the simple notion that $\mathrm{SN}$ Ia result form the transfer of hydrogen onto a white dwarf in a binary system, depending on the rate of mass transfer (Iben and Tutukov 1984; Wheeler 1992). In brief, at low transfer rates one expects a nova explosion that at least expels most of the accreted hydrogen back into space, and may even remove some of the white dwarf, reducing not increasing, its mass. At more moderate transfer rates the heat of accretion prevents the hydrogen from being degenerate, but a thick layer of degenerate helium is formed that detonates and gives entirely the wrong composition of the ejecta. At yet higher rates, both the hydrogen and helium are burned non-degenerately, but the requirement that there be two stable burning shells on the progenitor demands very bright progenitors and it is not obvious that any candidate systems are observed. At the highest transfer rates, the white dwarf cannot assimilate the new material, and a common envelope may form which would contaminate the explosion with hydrogen in an unacceptable way. Thus, given the assumptions of the quantitative studies to date, all transfer rates of hydrogen from near zero to infinity have been ruled out, for one reason or another.

As discussed in Wheeler (1992), one or more of these arguments may be incorrect and it is very important to critically reexamine them. As an example, let us look at the cataclysmic 
system depends on angular momentum loss. If magnetic braking dominates, then the orbit should shrink as mass is transferred and the white dwarf will evolve down the nova/dud line to smaller masses. If magnetic braking is not the panacea that it is sometimes portrayed to be (Shafter, Wheeler, and Cannizzo 1986; Wamer 1987) and if nuclear evolution dominates in a significant fraction of the systems, then mass transfer could be approximately conservative and the white dwarf will grow in mass. Wheeler (1992) argues that the evolution could lead to very conducive conditions for the central ignition of carbon and a suitable explosion of a SN Ia. Clearly this is not the final word in this regard, but this discussion illustrates that the question of triggering a SN Ia by transfer of hydrogen is not dead.

\section{General Tests for Progenitor Evolution}

Given a number of possible progenitor systems and the large theoretical uncertainty that attends various evolutionary scenarios and estimates of supernova rates, it is of great importance to determine if there are new observational constraints that could be brought to bear on the issue.

Wheeler (1992) points out that any binary evolution scenario involving mass transfer is unlikely to be perfectly conservative. The mass transfer process should be sloppy and the left overs from such a process might give important clues to the progenitor evolution. If there is a circumstellar nebula around a SN Ia, then it might absorb or fluoresce in optical or UV lines and reveal its presence and nature. By example, the circumstellar nebula surrounding SN 1987A showed fluorescence resulting from the UV burst at shock breakout that was the major argument that the progenitor had recently been a red supergiant. The circumstellar nebula around a SN Ia is likely to be dilute, given the lack of evidence for associated radio emission, but the UV pulse should be especially intense and proper observations might yield some clue to the surrounding matter.

Various configuration are possible. A common envelope phase could leave a surrounding shell or ring of hydrogen. If the progenitor is a cataclysmic variable, then a wind of hydrogenrich matter could be generated. If the progenitor were a double degenerate system, the circumstellar matter could be almost pure carbon and oxygen. It would be worthwhile to construct theoretical models to define the possible observable properties of such matter and then search for it.

In fact, there may already be observational evidence for a hydrogen nebula. The most recent and best evidence is given by Polcaro and Viotti (1991). They observed a narrow, $460 \mathrm{~km} \mathrm{~s}^{-1}$, absorption of $\mathrm{H} \alpha$ in a high resolution spectrum of SN 1990M about four days prior to maximum light. The feature was also seen in lower resolution data a day earlier but was barely discemable three days later. The evidence for an absorbing layer of hydrogen seems very clear, but the data show that the effect is transient. The velocity is too high to be associated with the interstellar medium, but far too low to be associated with the ejecta of the supernova itself. It thus seems to arise in a circumstellar layer previously ejected from the star.

Polcaro and Viotti estimate a column depth in the $n=2$ level of hydrogen to be $5.4 \times 10^{12} \mathrm{~cm}^{-2}$, but an estimate of the total column depth of the layer is difficult because it depends on the ratio of hydrogen in the neutral and first excited state, which is very temperature sensitive. Polcaro and Viotti suggest that the event is transient because the surrounding matter is swept up by the supernova ejecta. They conclude the matter is very close to the supernova, $\sim 10^{15} \mathrm{~cm}$. Alternatively, the matter could be ionized away by the UV burst and be at a distance of about 15 
light days, or $\sim 4 \times 10^{16} \mathrm{~cm}$. A UV burst of ergs would be sufficient to ionize about $3 \mathrm{M}_{\odot}$ worth of hydrogen, suggesting an upper limit to the amount of circumstellar matter.

Polcaro and Viotti point out that the observed velocity is too high for a red giant and too low for a very hot star, thus providing a potentially useful constraint on the progenitor system. It should be borne in mind, however, that the dynamics could be complex and the velocity not simply identified with normal single star behavior. For instance, matter could flow off a red giant in a wind of $10 \mathrm{~km} \mathrm{~s}^{-1}$ but then be exposed to the radiation flux or even a high speed wind from the compact companion. The result might be to accelerate the flow to the observed velocity.

Polcaro and Viotti also estimate that if the circumstellar matter is shed from a common envelope, that process must have occurred only ten years before the explosion, implying that the double degenerate stars were born only $10^{10} \mathrm{~cm}$ apart. If anything, Polcaro and Viotti seem to have minimized the problems with this hypothesis. At $460 \mathrm{~km} \mathrm{~s}^{-1}$, the envelope could have expanded for only $0.6 \mathrm{yr}$ if it were at $10^{15} \mathrm{~cm}$ and $24 \mathrm{yr}$ if it were at $4 \times 10^{16} \mathrm{~cm}$. Equating this time with the subsequent time for orbital decay using equation 3 gives an initial orbital period of only $5-20 \mathrm{~s}$ for a total mass of $1 \mathrm{M}_{\odot}$, and hence an initial separation of $5-10 \times 10^{8} \mathrm{~cm}$. This is very extreme since the binding energy of the two cores at that point, $\sim 10^{50}$ ergs would greatly exceed the binding energy of several solar masses of common envelope at a radius of $>10^{12} \mathrm{~cm}$, which is $<10^{48}$ ergs. Formation at such a small orbital period would solve the problem of the failure to observe a significant number of double degenerates at large orbital period, but if this is the rule, then most SN Ia would have to be much younger than a Hubble time. It is thus not at all clear that the hydrogen observed by Polcaro and Viotti can be connected with a common envelope process.

Another clue that some circumstellar hydrogen attends SN Ia was given by Branch et al. (1983) in their analysis of SN 1981B. A spectrum on March 13, illustrated in Figure 2, showed what might have been a small emission feature at the position of $\mathrm{H} \alpha$. It was not seen on March 6 - 9, near maximum light, nor on March 18. Branch et al. concluded that while the reality of the feature could not be definitely established, it also could not be easily attributable merely to noise in the data. From arguments based on the recombination time after the passage of the UV burst, McCall (unpublished 1981) estimated that if the feature were real, the circumstellar matter would be about 20 light days from the supernova with a density greater than $5 \times 10^{-6} \mathrm{~cm}^{-3}$ and a mass in excess of $0.1 \mathrm{M} \odot$.

It is conceivable that these two observations are manifestations of the same phenomenon and the observation of Polcaro and Viotti gives more confidence that the feature reported by Branch $e t$ al. was real. If there were a shell around SN 1981B at $\sim 20$ light days, the ejecta would have hit it at $\sim 200-600$ days, but there were no observations then. SN 1981B did not show any evidence for $\mathrm{H} \alpha$ absorption near maximum, March $6-9$, but it was not observed prior to maximum. It is not clear that there were any observations of SN 1990M after maximum that might have detected a brief phase of $\mathrm{H} \alpha$ emission. SN $1990 \mathrm{~N}$ shows a weak absorption at about $6600 \AA$ in a spectrum obtained 7 days after B maximum which does not appear in other SN Ia spectra of the same epoch (Leibundgut $e$ t al. 1991). Other SN Ia spectra should be examined closely around maximum light for evidence of $\mathrm{H} \alpha$. Higher resolution spectra dedicated to this task are probably in order.

Other evidence for hydrogen in the spectra of SN Ia events could be sought in the outer layers of the exploding white dwarf or ablated from the companion. Wheeler and Harkness (1990) argue that if the hydrogen in the outer layers is in LTE then as much as $0.1 \mathrm{M}_{\odot}$ could be present without contributing significantly to the spectra near maximum light. Branch et al. (1991) have 
SN NGC4536 13MAR81 1981B

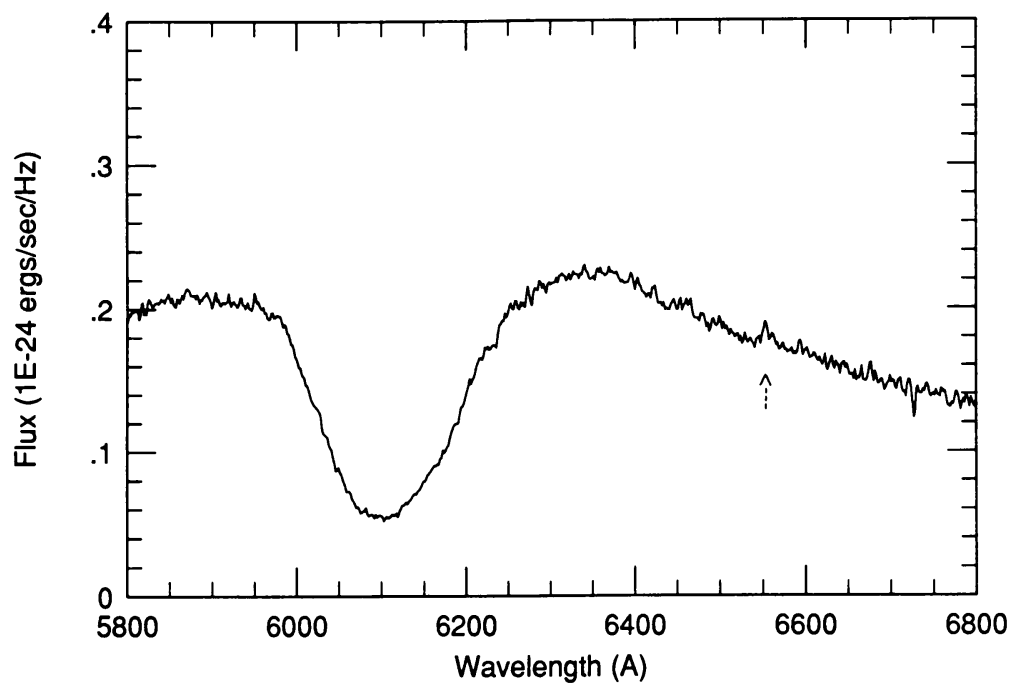

Figure 2. Spectrum of SN 1981B on March 13 about one week past optical maximum showing what may be a small $\mathrm{H} \alpha$ emission feature (Branch et al. 1983).

done an analogous calculation relaxing the assumption of LTE and conclude that the mass of such a layer spread uniformly throughout the line forming layer has an upper limit of $\sim 0.01 \mathrm{M}_{\odot}$.

Applegate and Ternan (1989) have reconsidered the problem of stripping and ablating hydrogen from a companion (cf. Lecar, Wheeler, and McKee, 1976), but they did not consider red giant companions and they make the implicit assumption that the stripped matter will be ejected at high velocities. Chugai (1986) has argued, however, that both the stripped and ablated matter will emerge behind the supernova ejecta at rather low velocities. This has been confirmed by two-dimensional dynamic calculations by Livne, Tuchman, and Wheeler $(1991,1992)$. The dynamic studies show that the leading edge of the shock wraps around the companion and continues with a high velocity so that the stripped matter lags the shock front and ejecta. Along the line of centers the ejecta heat the companion and then are reflected. The matter ablated from the companion from this heating again lags the reflected ejecta at lower velocities. The result is that the hydrogen removed from the companion will be contained within the supernova ejecta. The ejecta must then expand and cool and turn optically thin for this matter to be observable. The hydrogen thus revealed may be heated by radioactive decay in the ejecta and show up as nebular emission at later times. Such late time hydrogen emission can in principle be modeled to provide theoretical estimates of the strength of such features. Once again a close examination of nebular spectra of SN Ia should be made to seek any such emission. Good signal to noise data with 
decent resolution will be necessary to separate such features, e.g. $\mathrm{H} \alpha$ in emission, from the complex [Fe II] features that dominate at that phase.

\section{Summary and Discussion}

Despite all the uncertainties of the progenitor evolution and explosion mechanism, spectral analysis of SN Ia continues to strongly suggest that exploding carbon/oxygen white dwarfs represent the only viable model.

To make progress on the question of progenitor evolution more emphasis needs to be given to dedicated observational searches for relevant clues. More effort to detect transient $\mathrm{H} \alpha$ emission or absorption in many events is necessary and theoretical models of the expected effect of the strong UV burst on different configurations of circumstellar matter should be investigated. Continued effort should be made to both predict and search for any high velocity hydrogen representing the outer layers of the white dwarf. Likewise models should be made for the emission of any matter blasted from the companion that is most likely to be manifested in the later nebular phases and specific observational searches should be made for such matter.

The tantalizing evidence for hydrogen in circumstellar shells in some SN Ia events is difficult to rationalize with the double degenerate mechanism except under rather extreme circumstances. The obvious alternative is that the hydrogen is involved in some way with the mass transfer that brings the white dwarf to its explosive endpoint.

A critical issue remains to identify the progenitors of SN Ia. It is very difficult to believe that there is as yet some new, previously unobserved, class of binary stars that are the long sought progenitors. It seems much more likely that someone at this meeting is studying the progenitors but we have just not had the wits to recognize which they are. Kenyon (1986) and Wheeler (1992) have considered the symbiotic stars as possible progenitors. They are interesting candidates because they might represent the bright, but as yet not specifically identified, progenitors that are accreting fast enough to prevent degenerate ignition of both hydrogen and helium. The problem is that there does not seem to be a sufficient number of such systems. Given that current estimates of the Galactic SN Ia rate are somewhat less that some prior estimates, it might be worthwhile to examine the question of the production rate of symbiotic variables again. It would also be very useful to know the masses of the white dwarfs involved in those systems that have degenerate companions.

Another interesting class of stars discussed at this meeting are the barium stars. These can not be the direct progenitors of SN Ia because they are not undergoing mass exchange, but they are enticing because they are now widely thought to be systems containing white dwarfs and roughly solar mass $\mathrm{G}$ and $\mathrm{K}$ giant companions. These systems have undergone one stage of mass transfer as evidenced by the barium in the atmosphere of the giant companion. They have presumably somehow avoided dynamically unstable mass transfer and the common envelope phase. The important question in the current context is the nature of the next evolutionary stage when the giant companion ceases core helium burning and climbs the asymptotic giant branch. It is likely that this will trigger mass transfer. Clearly, it would be of great interest to explore whether the resulting evolution has a connection to symbiotic stars or SN Ia.

This research was supported in part by NSF Grant 8915754 and NASA Grant NAGW 1807. Numerical calculations were performed on the Cray Y-MP at the University of Texas System Center for High Performance Computing. 


\section{References}

Applegate, J. H. and Ternan, J. L. 1989, Ap. J., 340, 380.

Bath, G. T. and Shaviv, G. 1978, M.N.R.A.S., 183, 515.

Bragaglia, A., Greggio, L., Renzini, A., and D'Odorico, S. 1990, Ap. J. Lett., 365, L13.

Branch, D., Pauldrach, W. A., Puls, J., Jeffery, D. J., and Kudritzki, R. P. 1991, in SN1987A and Other Supernovae, eds. J. Danziger, W. Hillebrandt, F. Ferrini, and L. Lucy (Garching: ESO), in press.

Branch, D., Lacy, C. H., McCall, M. L., Sutherland, P. G., Uomoto, A., Wheeler, J. C., and Wills, B. J. 1983, Ap. J., 270, 123.

Chugai, N. 1986, Sov. Astron., 30, 563.

Foss, D., Wade, R. A., and Green, R. F. 1991, Ap. J., 374, 281.

Harkness, R. P. 1991a, in Supernovae, ed. S. E. Woosley (New York: Springer-Verlag), p. 454.

Harkness, R. P. 1991b, in SN1987A and Other Supernovae, eds. J. Danziger, W. Hillebrandt, F. Ferrini, and L. Lucy (Garching: ESO), in press.

Iben, I., Jr. and Tutukov, A. V. 1984, Ap. J. Suppl., 54, 335.

Iben, I., Jr. and Tutukov, A. V. 1992, in Proceedings of the McDonald Observatory 50th Anniversary Symposium, Frontiers of Stellar Evolution, ed. D. L. Lambert (Provo: Astronomical Society of the Pacific), in press.

Kenyon, S. J. 1986, The Symbiotic Stars, (Cambridge: Cambridge University Press).

Khokhlov, A. 1991, Astron. Ap., in press

Lecar, M., Wheeler, J. C., and McKee, C. F. 1976, Ap. J., 205, 556.

Leibundgut, B., Kirshner, R. P., Filippenko, A. V., Shields, J. C., Foltz, C. B., Phillips, M. M., and Sonneborn, G. 1991, Ap. J. Lett., 371, L33.

Livne, E., Tuchman, Y., and Wheeler, J. C. 1991, in Supernovae, ed. S. E. Woosley (New York: Springer-Verlag), p. 219.

Livne, E., Tuchman, Y., and Wheeler, J. C. 1992, in preparation.

Maza, J. and van den Bergh, S. 1976, Ap. J., 204, 519.

Patterson, J. 1984, Ap. J. Suppl., 54, 443.

Polcaro, V. F. and Viotti, R. 1991, Astron. Ap., 242, L9.

Robinson, E. L. and Shafter, A. W. 1989, Ap. J., 322, 296.

Saffer, R. A., Liebert, J. W., and Olszewski, E. W. 1988, Ap. J., 334, 947.

Shafter, A. W., Wheeler, J. C., and Cannizzo, J. K. 1986, Ap. J., 305, 261.

Shara, M. M., LIvio, M., Moffat, A. F. J., and Orio, M. 1986, Ap. J., 311, 163.

van den Bergh, S. 1990, PASP, 102, 1318

van den Bergh, S. and Tammann, G. A. 1991, Ann. Rev. Astron. Ap., 29, 363.

Warner, B. 1987, M.N.R.A.S., 227, 23.

Webbink, R. F. 1984, Ap. J. Lett., 277, 355.

Wheeler, J. C. 1990 in Supernovae, eds. J. C. Wheeler, T. Piran, and S. Weinberg (Singapore: World Scientific), p. 1.

Wheeler, J. C. 1992, in Proceedings of the McDonald Observatory 50th Anniversary Symposium, Frontiers of Stellar Evolution, ed. D. L. Lambert (Provo: Astronomical Society of the Pacific), in press.

Wheeler, J. C. and Harkness, R. P. 1990, Rep. on Prog. in Physics, 53, 1467.

Woosley, S. E. 1991, in Supernovae, eds. J. Audouze, S. Bludman, R. Mochkovitch, and J. Zinn-Justin (Paris: Elsevier), in press. 